\title{
Revistas de arquitetura, urbanismo, paisagismo e design: a divergência de perspectivas
}

\author{
H ugo Segawa \\ A rquiteto, professor livre-docente do D epartamento de Arquitetura e \\ U rbanismo da Escola de Engenharia de São Carlos da U niversidade \\ de São Paulo. Editor-regional do The Journal of Architecture do RIBA \\ (Londres). \\ E-mail segawahg@usp.br
}

\section{Adriana C rema}

Arquiteta, pesquisadora do Programa de Pós-Graduação em Arquitetura e Urbanismo do Departamento de Arquitetura e U rbanismo da Escola de Engenharia de São Carlos da U niversidade de São Paulo.

\section{M aristela G ava}

A rquiteta, pesquisadora do Programa de Pós-Graduação em Arquitetura e Urbanismo do Departamento de Arquitetura e U rbanismo da Escola de Engenharia de São Carlos da U niversidade de São Paulo.

\section{Resumo \\ Trata de estabelecer as características atuais de revistas de arquitetura, urbanismo, paisagismo e design publicados no Brasil para um possível enquadramento como periódicos científicos ou técnicos. Comenta uma metodologia corrente de avaliação de publicações científicas de outras áreas de conhecimento e contextualiza sua aplicação diante da natureza diversificada da produção científica da área de arquitetura e urbanismo.}

\section{Palavras-chave}

Metodologia para avaliação de periódicos; Periódicos científicos brasileiros; Periódicos de arquitetura e urbanismo; Periódicos de paisagismo; Periódicos de design.

Architecture, urbanism, landscape architecture and design periodicals: divergent perspectives

\begin{abstract}
This paper presents the features of Architecture, Urbanism, Landscape Architecture and Design periodicals published in Brazil for their potential consideration as scientific or technical periodicals. Comments a current methodology of evaluation for scientific periodicals from different fields of knowledge and the pertinence of its applicability due to the diverse nature of the scientific output from the Architecture and Urbanism area.
\end{abstract}

\section{Keywords}

Methodology for periodicals evaluation; Brazilian scientific periodicals; Architecture and urbanism periodicals;

Landscape architecture periodicals; Design periodicals.

\section{IN TRO DUÇÃO}

Ao percorrer a seção de revistas de uma boa biblioteca especializada em arquitetura, urbanismo, paisagismo e design (AUPD), ao mesmo tempo que um interessado encontrará uma coleção impressionante de publicações tratando de inúmeros aspectos em uma variedade de formatos, cores e acabamentos, um consulente com um olhar mais acadêmico terá dificuldade para identificar títulos que possam ser caracterizados como "periódicos científicos e técnicos", nos padrões consagrados em outras áreas de conhecimento. Todavia, entre um boletim noticioso corporativo, volumes com o porte de livros, revistas fashion, sisudas publicações ou magazines de arranjos interiores, há um conjunto com um rico repertório de informações e documentação de uma época. Sem arriscarmos variegar em infindáveis discussões epistemológicas e semânticas, vamos apenas constatar e reconhecer empiricamente a natureza específica da difusão da informação e da cultura em AU PD através de um periodismo que se ocupa tanto de um jornalismo de serviço como oferece densas e impenetráveis monografias. É uma história de pelo menos três séculos de duração na França, país em que pesquisadores sistematizaram um trabalho sobre o periodismo francês de arquitetura do século XVIII ao XX, e contabilizaram, entre 1800 e 1970, 606 revistas. ${ }^{1} \mathrm{O}$ u de um universo próximo e distante ao mesmo tempo, como o conjunto de 68 títulos em publicação na A mérica Latina em $1993^{2}$ e dos quais talvez tenhamos contato com não mais de uma dúzia deles. A o se consultar o U Irich's I nternational Periodicals Directory mediante a seleção de descritores buscan do caracterizar o conteúdo da revista, elaboramos a tabela 1, apenas de perfil quantitativo.

Periodismo multifacetado que se consolidou ao longo do século XX em uma cultura literária e visual que foi também responsável pela veiculação de idéias e imagens que ajudaram a transformar o ideário e a prática profissional, circunscrito naquilo que se convencionou chamar de arte, arquitetura e urbanismo modernos. 
Embora de forma lacônica, estas palavras iniciais apenas cogitam chamar a atenção da natureza distinta do periodismo em arquitetura e urbanismo diante das áreas de conhecimento que melhor normatizaram seus veículos impressos de difusão de conhecimento e memória com um rigor científico nem sempre compartilhado pelo universo criativo da arte, da arquitetura, do urbanismo, do paisagismo e do design em que pesem os esforços para nosso enquadramento na 'grande área' das ciências sociais aplicadas, conforme classificação do sistema Conselho $\mathrm{N}$ acional de Desenvolvimento Científico e Tecnológico (C N Pq) / Coordenação de A perfeiçoamento de Pessoal de Nível Superior (Capes) e da Fundação de Amparo à Pesquisa do Estado de São Paulo (Fapesp).

\section{A DIVERSIDADE CORRENTE}

Para auxiliar nessa distinção de natureza epistemológica que contempla a área de AU PD, uma comparação de sistemas tal vez nos auxilie na compreensão das dinâmicas específicas das áreas.

Setor de ponta da pesquisa científica brasileira, a área de medicina se vangloria de sua produtividade, respondendo por 14\% das citações brasileiras no exterior em 1998 e contribuindo com $0,5 \%$ da produção científica global. Sua base de dados é o mais avançado sistema de registro e intercâmbio de informação técnico-científica latinoamericano com a estruturação desenvolvida pelo C entro Latino-A mericano e do C aribe de Informação em Ciências da Saúde (Bireme). "Com o aprimoramento da avaliação dos programas de pós-graduação no país, tendo-se como critério essencial a relevância da produção intelectual, aferida pela qualidade dos veículos científicos, espera-se, em breve, um crescimento ainda maior", rejubilam-se os especialistas da área. ${ }^{3} \mathrm{~A}$ área de medicina no país conta com 165 programas de mestrado e 141 de doutorado, segundo a C apes (dados de setembro de 2003), ${ }^{4}$ números incomparáveis com a modéstia de nossa área: dezessete programas de mestrado e nove de doutorado (incluindo os dedesign $).^{5}$

Em muitas áreas, indicadores como o Science Citation A bstracts do ISI (Institute for Scientific Information) são referên cias fun damentais para a aferição de produtividade e, por suposto, de qualidade. $M$ as tais indicadores não são capazes de cobrir as variáveis que incidem na produção em AU PD. Entre uma experiência laboratorial ensaiando o comportamento físico de um material de interesse para o conforto ambiental à análise de impactos socioambientais de intervenções urbanas, passando por uma ref lexão sobre a autoria do risco de um chafariz do século XVIII em 0 uro Preto, não há parâmetros comuns
TABELA 1

Q uantificação de títulos localizados no U Irich's International Directory, conforme busca por descritores

\begin{tabular}{l|c}
\hline Descritor & $\begin{array}{c}\text { Número de títulos } \\
\text { localizados }\end{array}$ \\
\hline Architecture & 1.794 \\
U rbanism & 14 \\
U rban Planning & 1.529 \\
Landscape & 178 \\
Design* & 3.097 \\
Industrial Design & 32 \\
Housing** & 1.833 \\
\hline
\end{tabular}

* 0 descritor Design tem um alcance que extrapola o interesse da área e, sozinho, não é um parâmetro suficiente.

** $\mathrm{O}$ descritor Housing alcança publicações de muitas outras áreas.

que possam aferir tão distintos interesses e produtos científicos. A variedade de enfoques, procedimentos, aproximações e interpretações dos objetos tratados em AU PD dificultam caracterizar um sistema uniforme e monolítico do ponto de vista qualitativo e estatístico suficiente para estabelecer um sistema coerente e mensurável, como se identifica, por exemplo, na área de medicina.

\section{A NECESSIDADE DE QUALIFICAÇÃO}

A inerente dificuldade de avaliação de periódicos em AUPD não exime a área de estabelecer critérios qualitativos para publicações, dentro das premissas da geração, preservação, organização e difusão de conhecimento. É possível e desejável que a qualidade da informação seja objeto de preocupação de autores, publishers, editores, bibliotecários, arquivistase, naturalmente, pesquisadores eusuários das informaçõeseleitores em geral, equeesseconjunto de interessados estabeleça um sistema confiável de referência comum, por maior que seja o risco de se avalizar uma camisa-de-força.

Esforços nesse sentido têm sido desenvolvidos e uma indicação indispensável nesse âmbito é o trabalho "A valiação de periódicos científicos e técnicos brasileiros" de Rosaly Favero Krzyzanowski e M aria Cecília Gonzaga Ferreira, ${ }^{6}$ que "trata da metodologia desenvolvida para avaliação conjunta de mérito (conteúdo) e desempenho (forma) dos periódicos científicos e técnicos correntes brasileiros." 0 modelo elaborado por essas bibliotecárias foi adotado em uma proposta de avaliação para a Capes na subárea de psicologia. ${ }^{7}$ 


\section{Revistas de arquitetura, urbanismo, paisagismo e design: a divergência de perspectivas}

0 trabalho de Krzyzanowski \& Ferreira é significativo por tentar estabelecer parâmetros gerais a partir de uma amostragem que conformou a base das proposições dos modelos para análise de periódicos científicos, com hipóteses de aplicação nas 'grandes áreas' de engenharias, exatas, biológicas e humanas. $M$ as, para a nossa área, a inerente heterogen eidade de perspectivas obriga a assimilar conteúdos e metodologias dessas áreas. $\mathrm{N}$ enhum dos modelos isoladamente contemplaria satisfatoriamente 0 arco de conhecimentos pretendidos em AUPD. Os modelos propostos organizam uma primeira referência para a elaboração de um roteiro para 0 exame das revistas, mas devem ser revistos em face das peculiaridades da área.

\section{O CONTEXTO DAS PUBLICAÇÕES}

A pesar dos evidentes contrastes entre o sistema de publicações em AUPD no exterior e no Brasil, nos principais centros se identifica um problema preocupante, conforme anota o editorial de William S. Saunders, da revista GSD N ews, publicada pela $\mathrm{H}$ arvard U niversity Graduate School of Design, em edição especial sobre design publishing em 1996:

Nosso tópico em destaque - publicação em design, particularmente revistas - tem sofrido grandes mudanças nos últimos anos, pode-se dizer para pior. M odestas publicações intelectuais (incluindo as estudantis) lutaram ou não lograram sobreviver. Entre os 21 editores ou exeditores que comentam sobre publicações de design nesta edição, cinco deixaram seus postos há pouco tempo, aparentemente porque seus publishers desejavam revistas mais lucrativas e menos críticas.

U m freqüente comentário nas páginas que se seguem é que as publicações de design têm se voltado cada vez menos para a crítica rigorosa e honesta de projetos de arquitetura, urbanismo e paisagismo contemporâneos, e cada vez mais o que se publica é promocional ou simples documentação. A ausência de crítica vigorosa produz um ambiente no qual designers são menos autocríticos, menos cientes da natureza de suas próprias atividades projetuais e, portanto, menos propensos para produzir trabalhos de alta qualidade?

0 depoimento, na mesma edição, de $\mathrm{V}$ ittorio $\mathrm{G}$ regotti editor de C asabella por 14 anos - também é el oqüente:

Nos últimos 30 anos, testemunhamos o declínio das tradições do jornalismo arquitetônico que data da virada do século. Por muitas décadas e até recentemente, as revistas de arquitetura eram os foros de discussões passionais e partidárias; quase sempre elas mantiveram uma forte e precisa posição sobre a disciplina arquitetônica.

No entanto, nas recentes três décadas, revistas de arquitetura o contentamento de se tornarem meros espelhos da profissão: elas preferiram simplesmente registrar, geralmente de uma maneira plural e neutra, 0 que estava acontecendo. 0 dúbio resultado dessa transformação tem sido, de um lado, o aumento da quantidade de material publicado e, de outro, o embaçamento do critério pelo qual este material é selecionado. Esta ten dência vem acompanhada também do incremento da "profissionalização" da imprensa de arquitetura, evidenciado no crescimento da complexidade estrutural da equipe editorial e a elevação dos custos de publicação. ${ }^{9}$

Gregotti está aludindo (entre outros pontos) à chamada "revista de tendência", na qual o conteúdo editorial ref lete orientações arquitetônicas definidas, como as que caracterizaram as querelas entre modernos e acadêmicos e a consolidação da arquitetura moderna (L'A rchitecture D 'Aujourd'H ui) no segundo quartel do século XX, a crise da modernidade no imediato pós-Segunda Guerra (C asabella-C ontinuità, A rchitectural Review), ou a questão pós-moderna nos anos 1970/ 80 (A rchitectural D esign).

No Brasil, revistas como H abitat eM ódulo dos anos 1950 eA crópoledos anos 1960 (com menos rigor) aproximaramse das linhas editoriais de tendência, como Arquitetura ref letiu as posições da corporação nessa mesma década, até o fenecimento da imprensa de arquitetura no início dos anos 1970. 0 ressurgimento das publicações regulares nos anos 1980, com a Projeto (a partir de 1979) e AU (desde 1985), não marcou a retomada de "revistas de tendência", mas refletiu as incertezas de um país no limiar da redemocratização, o atordoamento pósmoderno e a concordata da modernidade brasileira.

A referência à "profissionalização" por G regotti também assola 0 exíguo campo editorial brasileiro. A legislação estabelece para periódicos a obrigatoriedade de um jornalista como responsável. N ossas revistas de maior circulação são editadas e redigidas por jornalistas ainda in experientes de conhecimentos em arquitetura. Essas publicações se ressentem de um conselho editorial consistente ou atuante. As matérias sobre projetos arquitetônicos se limitam a descrições de técnicas construtivas ou declarações textuais reproduzindo acriticamente os discursos dos autores das realizações. São econômicas em análises ou opiniões, raramente trazendo comentários críticos ou enveredando por relações conceituais - salvo quando há freelancers 


\section{Hugo Segawa / Adriana C rema / Maristela Gava}

especialistas, geralmente com escritos reduzidos a um boxe. Os poucos jornalistas experientes, que cultivaram algum domínio na área, não mais atuam regularmente nesses veículos. Edições recentes (primeiro semestre de 2002) trazem conteúdos em que a parte editorial não se diferencia claramente da comercial. No outro extremo, as rarefeitas revistas acadêmicas pecam pelo amadorismo na dinâmica de redação, da produção e circulação dos periódicos.

\section{CATEGORIAS DE PUBLICAÇÕES}

À medida que se reconhece a variedade como um atributo inerente ao periodismo em AU PD, parecenos sensato estabelecer uma categorização de revistas para que se possa articular um procedimento de aferição compatível com as diferentes características editoriais, flexibilizando a rigidez que codifica um típico "periódico científico e técnico."

$\mathrm{N}$ ão temos conhecimento de classificação sistemática de revistas em AUPD que busque demarcar perfis no sentido de aferir sua qualidade. Ramón Gutiérrez \& $M$ arcelo $M$ artín, na já citada bibliografia latino-americana de revistas, ${ }^{10}$ estabelecem uma ordem de agrupamento que, embora não explicitando sua razão, insinua um critério editorial. As revistas são classificadas com as seguintes rubricas:

- revistas de edição universitária (sob responsabilidade de escolas de arquitetura ou programas de pós-graduação);

- revistas comerciais (publicações de editoras privadas);

- revistas de centros de pesquisa, organizações nãogovernamentais, instituições não-acadêmicas e outras.

- revistas de agremiações profissionais;

- cadernos de arquitetura em jornais diários (não existem no Brasil).

Considerando aspectos de mérito (conteúdo) e desempenho (forma) que comentaremos adiante, propomos uma primeira aproximação quanto à natureza dos periódicos a merecer avaliação, levando em conta a necessidade de abrigar a heterogeneidade da área e a realidade editorial brasileira como variáveis que modelam tanto o conteúdo quanto a forma:

1. periódicos voltados predominantemente à publicação de trabalhos originais derivados de investigações desenvolvidas em centros ou grupos de pesquisa, programas de pós-graduação, instituições independentes e a produção intelectual, artística, arquitetônica, urbanística, paisagística e de design de profissionais de reconhecida credibilidade entre os pares;

2. anais de encontros científicos ou profissionais contendo a publicação completa de conferências, comunicações, mesas-redondas e painéis, promovidos por instituições de reconhecida credibilidade e consolidação no meio acadêmico ou profissional, ou eventos pontuais organizados com todos os requisitos técnicos e científicos que atribuam equivalência qualitativa a eventos periódicos;

3. revistas especializadas que, mesmo não cumprindo aspectos formais de qualificação acadêmica, são reconhecidas no meio como veículos de práticas, idéias, proposições, inovações e reflexão continuadas, caracterizando-se como fontes de consulta ou atualização do estado-da-arte da produção recente;

4. revistas, jornais, cadernos culturais e técnicos de diários e publicações nãoespecializados e de circulação ampla ou reconhecimento qualitativo nacional ou internacional, voltados à publicação de artigos, ensaios, análises, comentários e resenhas significativos para a difusão e 0 debate de aspectos correlatos à área e que ampliem a audiência dos temas para um público amplo e leigo;

5. revistas de arquitetura, arte, construção e decoração dirigidas a público leigo, que possam constituir repertório informacional da produção recente na área;

6. portais e páginas de Internet especializados na área.

A pesar de o Currículo Lattes aceitar produção científica e técnica difundida pela rede virtual, não vamos tratar, por enquanto, do último tópico - as revistas virtuais -, pela complexidade que envolve a consistência de conteúdos pela Internet.

\section{CRITÉRIOS DE AVALIAÇÃO}

Como procedimento geral, vamos tomar a metodologia apresentada por Krzyzanowkski \& Ferreira como referência que paira sobre as diversas áreas como um possível modelo. A dotando-se a sistemática de avaliação conjunta de mérito (conteúdo) e desempenho (forma), vamos tentar mediar uma metodologia de perfil genérico com a realidade corrente do periodismo em AU PD e estabelecer um refinamento crítico quanto à aplicabilidade de critérios possivelmente válidos em várias áreas, mas não necessariamente eficazes em nossa área. À medida que se estabeleça um juízo qualitativo sobre esses critérios, será possível discernir as propriedades de um esquema de pontuação como propõem as bibliotecárias. 
A itemização adiante não obedece estritamente à ordenação proposta por K rzyzan owkski \& Ferreira. N ossa análise também procurou comparar e compreender procedimentos de admissão de periódicos em bases de dados, e consultamos indexações de outras áreas: M edline (periódicos da área de Saúde), Lilacs (Literatura Latinoamericana e do Caribe em Ciências da saúde) e o SciELO (Scientific Electronic Library 0 nline). A ordenação apresentada ref lete tópicos que assimilam questões observadas nas bases de dados.

\section{AVALIAÇÃO DE MÉRITO}

1) Perfil da Publicação

U ma análise da estrutura geral de um periódico deveria contemplar os seguintes aspectos:

- ter predomínio de artigos originais (deverá ser feita uma avaliação por pares);

- publicar artigos predominantemente no núcleo da área;

- publicar trabalhos de autores de várias instituições, com abrangência estadual, nacional e internacional, em colaboração ou não;

- conter seções editoriais definidas; incluir editoriais, resenhas, documentos, relatos, cartas, entrevistas, depoimentos, bibliografias;

- não evidenciar interesses econômicos e/ ou conteúdo publicitário ou patrocínio comercial nos trabalhos publicados;

- não levantar dúvidas quanto à objetividade do material publicado.

2) Q ualidade do conteúdo

Os trabalhos devem ser examinados sob os seguintes aspectos:

- atualidade;

- originalidade;

- validade científica;

- importância, repercussão;

- identificação com a orientação temática da revista;

- contribuição analítica e interpretativa;

- abordagem de aspectos filosóficos, éticos ou sociais relacionados à área.
3) Comissão ou corpo editorial, conselho editorial

A existência de uma comissão ou corpo editorial constitui uma referência para o gerenciamento científico do periódico, bem como o conselho editorial é um atestado de credibilidade e confiabilidade da política editorial da revista. Como tal:

- a composição do conselho editorial deve ser pública e estável;

- devem ser especialistas reconhecidos, de origem nacional e internacional, e identificados quanto à origem institucional e nacionalidade. Deve-se evitar 0 predomínio de membros de uma instituição ou região geográfica;

- quando necessário, dever-se-á verificar a produção científica dos membros do conselho editorial em bases de dados especializadas.

\section{4) Seleção dos trabalhos publicados}

A revista deve especificar formalmente qual 0 procedimento para a aprovação de artigos. A arbitragem pode ser processada exclusivamente pelo conselho editorial, por consultores externos ou por ambos. A qualificação da revista é proporcional ao procedimento adotado.

\section{5) Bases de dados, indexação}

A indexação dos títulos em bases de dados internacionais é um critério corrente para a qualificação das publicações. Todavia, pesa o obstáculo da língua, porquanto o português não merece muita consideração nessas bases de dados. No âmbito da AU PD, há enorme dispersão da inserção de periódicos nos diferentes bancos que acolhem as especialidades. Temas como estudos urbanos, planejamento territorial e urbano e habitação são registrados em indexações na área de ciências sociais - Social Sciences Citation Index, Sociological A bstracts, Public A ffairs Information Service, A pplied Social ScienceIndex and A bstracts, International Bibliography of Sociology, SageU rban Studies A bstracts, C urrent C ontents/ Social \& Behavioral Sciences; os temas pertinentes à ciência e tecnologia são indexados no Science Citation Index; os de arte e de história no segmento específico - Arts and Humanities Citation Index, Bibliography of the H istory of Art, C urrent Contents/ Arts and H umanities, Art Index; a arquitetura e urbanismo em poucas bases, quese saibam - Architectural Periodicals Index (API), AveryIndex to A rchitectural Periodicals e o já mencionado Art Index. N esse conjunto de bases de dados (que não esgota as bases internacionais), salvo 
exame mais minucioso, a solitária publicação brasileira representada éa revista Projeto no A rchitectural Periodicals Index, por um motivo fortuito. Q uando participantes da equipe editorial da revista, na segunda metade dos anos 1980, H ugo Segawa e Ruth Verde Zein iniciaram uma rede de contatos internacionais que estabeleceu intercâmbio com a biblioteca do RIBA em Londres, responsável pela elaboração do API. O Ulrich's International Periodicals Directoryregistra que aProjeto é indexada na A very Index to A rchitectural Periodicals, enão no API. E o API registra apenas a publicação de projetos arquitetônicos e desprezou os textos, como a extinta seção "Ensaio \& Pesquisa", que era a editoria científica na Projeto.

A única base de dados de arquitetura no Brasil é o Índice de Arquitetura Brasileira, elaborado pela Biblioteca da Faculdade de Arquitetura e U rbanismo da U niversidade de São Paulo. Considerando um conjunto variável de publicação ao longo de meio século (revistas de 1950 até o presente), seu repertório de periódicos indexados é um sensível indicador do dinâmico quadro editorial brasileiro da segunda metade do século XX. 0 desenvolvimento e 0 aperfeiçoamento do Índice de A rquitetura Brasileira é uma resolução fundamental para o futuro da pesquisa em AU PD no Brasil.

\section{6) Indicadores de impacto}

$\mathrm{N}$ ão há sistemas abrangentes de avaliação de impacto como o Journal Citation Reports do ISI, medição com base em citações no conjunto de títulos da área. Esquemas alternativos, nem todos devidamente sistematizados, podem servir como referência neste quesito:

- número de bases de dados e indexadores nacionais e internacionais em que figurar a revista; inclusão em bibliografias, abstracts, sumários correntes impressos ou em CD-ROM ;

- indicador de uso do periódico medido pela estatística de retiradas do periódico do acervo ou consulta da publicação no recinto da biblioteca (medição existente em algumas das bibliotecas do SIBi/ U SP). Este indicador é fator relativo na avaliação de mérito;

- existência de coleção nas bibliotecas-base do sistema Comut. Este indicador também é relativo na avaliação de mérito.

Ci. Inf., Brasília, v. 32, n. 3, p. 120-127, set./dez. 2003

\section{AVALIAÇÃO DE FORMA}

\author{
1) N ormalização
}

O s periódicos científicos são orientados por normas nacionais ou internacionais (ABNT, ISO) cujas padronizações nem sempre podem ser ou são respeitadas por publicações da área de AU PD que não ostentam caráter propriamente acadêmico, mas encerram conteúdos significativos que devem ser considerados não pela forma, mas pelo mérito em uma avaliação de periódicos. Quesitos básicos geralmente são contemplados nos periódicos não-acadêmicos, mas a normalização científica não é parte usual da imprensa de arquitetura mundial. $\mathrm{N}$ a especificidade das publicações de caráter eminentemente científico, as referências são:

- especificar instruções para os autores quanto à normalização, formatação de textos e apresentação de originais;

- explicitar a normalização das referências bibliográficas;

- identificar a filiação do(s) autor(es) e das fontes de financiamento;

- ter registro no ISSN (International Standard Serial N umber) - item obrigatório;

- sumário na língua original ou bilíngüe;

- conter resumos e descritores dos trabalhos no idioma do texto original ou bilíngüe;

- anunciar explicitamente a periodicidade;

- indicar as principais datas do processo de arbitragem, inclusive as datas de recepção, revisões e aprovação.

2) Periodicidade, pontualidade, duração

A periodicidade e pontualidade na edição são pontos críticos nas publicações brasileiras em AU PD. A fora revistas com amparo comercial de mercado (patrocínios publicitários, às vezes institucionais), a maioria das revistas da área padece de problemas crônicos de financiamento que comprometem a regularidade da circulação e até mesmo a sua subsistência. Raros são os periódicos não-comerciais que não sofreram atrasos, interrupções ou alteraram a periodicidade. É um critério problemático para a área. Os programas de apoio a publicações científicas (CN Pq, Fapesp) são rigorosos neste quesito para a concessão de recursos, caracterizando um círculo vicioso. 


\section{Revistas de arquitetura, urbanismo, paisagismo e design: a divergência de perspectivas}

\section{3) Difusão}

As formas de distribuição constituem fator problemático entre os periódicos especializados e científicos e espel ham com mais ênfase a precariedade da circulação e consumo de revistas em geral no mercado editorial brasileiro. A especificidade dos conteúdos reduz em muito a audiência das nossas publicações para venda avulsa. M esmo algumas revistas de maior tiragem, com patrocínio comercial, não são distribuídas em bancas. A circulação dirigida, por assinatura, não tem vigência entre as publicações acadêmicas, sobretudo pelo fantasma da falta de regularidade e duração, tampouco uma circulação internacional, pela barreira do idioma. Restam a permuta e doação, formas consideradas de menor valor na avaliação dos periódicos, mas que constituem a regra no âmbito das nossas publicações de perfil científico. Há de se relativizar este critério diante da realidade brasileira.

O Portal de Periódicos Capes (www.periodicos.capes. gov.br) é limitado pelo número de publicações disponíveis on-line. $\mathrm{A}$ área de arquitetura e urbanismo abriga 24 revistas, a de planejamento urbano e regional, 36 revistas, e a de desenho industrial, oito revistas, totalizando 68 indicações, que na realidade somam 61 títulos, porque há revistas compartilhadas entre as áreas. $\mathrm{N}$ enhuma das revistas é editada no Brasil. ${ }^{11}$

\section{4) Alcance}

A qualificação de uma revista deve ser medida em função do público atingido, proporcional quanto à circulação e acolhimento institucional, estadual, nacional e internacional.

\section{PROV IDÊNCIAS}

$\mathrm{N}$ enhuma coleção de critérios terá sentido, caso não se estabeleça uma norma sistemática e consensual de confronto entre avaliação e avaliado e, principalmente, de avaliador e avaliado. Para tanto, parece-nos fundamental a constituição de um comitê técnico responsável pela elaboração de uma con duta normalizada em busca da qualificação dos periódicos em AU PD. I sto poderia ser organizado pela área de arquitetura e urbanismo e, em uma hipótese mais avançada, por uma associação nacional de pós-graduação ou pesquisa em arquitetura e urbanismo.

Parecenos importante também reforçar iniciativas já organizadas de indexação científica, como 0 desenvolvimento e aperfeiçoamento do Índice de
Arquitetura Brasileira, em elaboração desde a década de 1960 pela Biblioteca da Faculdade de Arquitetura e U rbanismo da U SP e que ora se propõe a iniciar um esforço de digitalização do índice para consulta on-line.

Embora fora do escopo deste trabalho, acreditamos que 0 investimento em revistas virtuais possa constituir uma saída razoável diante das dificuldades imediatas de consolidação de um mercado editorial de publicações em AUPD no Brasil, desde que considerados critérios de qualificação de conteúdo. Bibliotecas virtuais como a BVS, sob o cuidado da Bireme, são hipóteses a se considerar com atenção. A implantação do Portal de A rquitetura e U rbanismo pela Escola de Arquitetura e U rbanismo da U niversidade Federal Fluminense (www.prossiga.br/ uff.ndc/ arquitetura), lançado em abril de 2003, no âmbito do Programa Prossiga, é um sinal auspicioso nesse sentido.

A reconhecida experiência do portal Vitruvius (www.vitruvius.com.br) éuma demonstração plausível de circulação de informações de grande aceitação no meio acadêmico, apesar de não seguir inúmeros critérios de avaliação de mérito, como anteriormente comentado.

A cima de qualquer prescrição classificatória, 0 reconhecimento das iniciativas de aferição supõe um difícil consenso entre os pares sobre os muitos aspectos controversos que uma avaliação de periódicos científicos e técnicos pode evocar. E não se trata de uma dificuldade restrita à subárea de arquitetura e urbanismo.

\section{AGRADECIMENTOS}

A gradecemos os comentários e subsídios de A bílio G uerra (editor do portal Vitruvius), Diva Carraro de Andrade (Biblioteca da FFLCH / U SP), Eliana de Azevedo $M$ arques (Biblioteca da FAU / U SP), Paulo J. V. Bruna (excoordenador da área, C apes), Ruth V erdeZein (ex-editora adjunta da revista Projeto), Teresinha Coletta (Biblioteca da EESC / U SP), Vicente W issen bach (ex-editor das revistas Projeto e Finestra/ Brasil). As bibliotecárias Elena Luzia Palloni Gonçalves e Rosana Alvarez Paschoalino, da EESC/U SP, buscaram informações importantes para o conteúdo deste trabalho. 


\section{Hugo Segawa / Adriana C rema / Maristela Gava}

\section{REFERÊN CIAS}

1. LENIAU D, Jean-M ichel, BO U VIER, Béatrice. $(0 \mathrm{rg}$.). Les périodiques d'architecture, XVIII eXXesiècle: recherched'une méthode critique d'analyse. Paris : École des Chartes, 2001. Cit. in L'A rchitecture D'Aujourd'H ui, n. 337, p. 12, nov./ dec. 2001

2. GUTIÉRREZ, Ramón, M ARTÍN, M arcelo. Bibliografía iberoamericana de revistas de arquitectura y urbanismo. Madrid : Instituto Español de A rquitectura, U niversidades de Alcalá y Valladolid, 1993.

3. MARI, Jair de Jesus; ZAGO, Marco Antonio. O Brasil no cenário científico. Folha de S. Paulo, 31 maio 2002, p. A3.

4. COORDENAÇÃO DE APERFEIÇOAMENTO DE PESSOAL DO NIVEL SU PERIOR (Brasília, DF). M estrados/ doutorados reconhecidos. Disponível em: বhttp:// www.capes.gov.br>. A cesso em: 08 set. 2003.

5. Idem.
6. KRZYZA N OW SKI, Rosaly Fávero, FERREIRA, Maria Cecília Gonzaga. A valiação de periódicos científicos e técnicos brasileiros. Ciência da Informação, v. 27, n. 2, p. 165-175, maio/ago. 1998.

7. SABADINI, Angélica Z. Paulovic; SAM PAIO, M aria Imaculada Cardoso. A avaliação das revistas científicas em psicologia. [Brasília], CAPES, [1999?].

8. SAUNDERS, William S. From the editor. GSD N ews, Summer 1996, p. 2.

9. GREGOTTI, Vittorio. Rebuilding the rules. GSD News, Summer 1996, p. 30.

10. GUTIÉRREZ, Ramón; MARTín, Marcelo. Bibliografía iberoamericana de revistas de arquitectura y urbanismo. [S. I. : s. n. 1999?]. cit., passim.

11. Disponível em: <http://www.periodicos.capes.gov.br.>. A cesso em: 08 set. 2003. 